\title{
Down-regulation of SOSTDC1 promotes thyroid cancer cell proliferation via regulating cyclin A2 and cyclin E2
}

\author{
Weiwei Liang ${ }^{1, *}$, Hongyu Guan ${ }^{1, *}$, Xiaoying $\mathrm{He}^{1}{ }^{1}$, Weijian $\mathrm{Ke}^{1}$, Lijuan $\mathrm{Xu}^{1}$, Liehua \\ Liu ${ }^{1}$, Haipeng $\mathrm{XiaO}^{1}$ and Yanbing $\mathbf{L i}^{1}$ \\ ${ }^{1}$ Department of Endocrinology and Diabetes Center, The First Affiliated Hospital of Sun Yat-sen University, Guangzhou, \\ Guangdong, China \\ * These authors have contributed equally to this work
}

Correspondence to: Yanbing Li, email: easd04lyb@126.com

Keywords: SOSTDC1, thyroid cancer, proliferation, cyclin A2, cyclin E2

Received: February 26, $2015 \quad$ Accepted: August 13, $2015 \quad$ Published: September 10, 2015

This is an open-access article distributed under the terms of the Creative Commons Attribution License, which permits unrestricted use, distribution, and reproduction in any medium, provided the original author and source are credited.

\section{ABSTRACT}

Sclerostin domain containing protein 1 (SOSTDC1) is down-regulated and acts as a tumor suppressor in some kinds of cancers. However, the expression pattern and biological significance of SOSTDC1 in thyroid cancer are largely unknown. We demonstrated that SOSTDC1 was significantly down-regulated in thyroid cancer. Ectopic over-expression of SOSTDC1 inhibited proliferation and induced G1/S arrest in thyroid cancer cells. Moreover, SOSTDC1 over-expression suppressed the growth of tumor xenografts in nude mice. We also found that elevated SOSTDC1 led to inhibition of cyclin A2 and cyclin E2. Together, our results demonstrate that SOSTDC1 is down-regulated in thyroid cancer and might be a potential therapeutic target in the treatment of thyroid cancer.

\section{INTRODUCTION}

Thyroid cancer is currently the most common endocrine malignancy, and its incidence is rising fast [1]. Thyroid neoplasia is histologically classified into differentiated papillary carcinoma (PTC), follicular carcinomas (FTC) and undifferentiated anaplastic carcinoma (ATC). Molecular genetic studies have demonstrated that thyroid carcinogenesis involves gradual accumulation of a series of genetic and epigenetic alterations, leading to gain-of-function mutations in oncogenes and loss-of-function mutations in tumor suppressor genes [2, 3]. Consequently, there is a need for exploration of the genetic events involved in thyroid cancer initiation and progression to improve our understanding of thyroid tumorigenesis and develop effective therapeutic strategies.

SOSTDC1 (sclerostin domain containing protein 1, also known as WISE, USAG1, ectodin) functions as antagonist of bone morphogenetic proteins (BMP) and Wnt signaling [4]. SOSTDC1 participates in multiple developmental processes including the teeth [5], hair [6], limb [7] and head [8]. SOSTDC1 also plays a vital role in the development of various cancers, such as breast cancer [9], renal tumors [10], Wilms tumor [11] and gastric cancer [12]. For example, it has been reported that SOSTDC1 is significantly down-regulated in clear cell renal carcinoma and over-expression of SOSTDC1 inhibits proliferation of clear cell carcinoma cells through regulating both BMP and Wnt signaling [10]. Recently, Gopisetty et al. found that SOSTDC1 was down-regulated in gastric tumors, and acted as a tumor suppressor in gastric cancer [13]. However, the expression and biologic function of SOSTDC1 in thyroid cancer remains unclear.

The retinoblastoma tumor suppressor protein $(\mathrm{pRb})$, which has been identified as a human tumor suppressor, plays an integral role in the regulation of cancer cell cycle progression [14-16]. pRb controls the G1/S boundary, in large part by repressing the transcriptional activity of the E2F transcription factors, which binds with pRb. Many growth factors lead to a significant phosphorylation of $\mathrm{pRb}$, resulting in its inactivation. Inactivated $\mathrm{pRb}$ can release and active E2Fs. Free E2Fs function as transcription factors can induce the expression of many genes involved in G1/S transition [17]. However, little is known regarding the function and regulation mechanisms of $\mathrm{Rb}-\mathrm{E} 2 \mathrm{~F}$ pathway in thyroid cancer.

In this study, we show that SOSTDC1 is downregulated in thyroid cancer. Moreover, our data suggest 
that SOSTDC1 inhibits the proliferation of thyroid cancer cells via regulation of cyclin A2 and cyclin E2.

\section{RESULTS}

\section{SOSTDC1 is clinically associated with the Rb-E2F pathway}

We analyzed the expression of SOSTDC 1 in 51 pairs of thyroid tumors and their adjacent non-tumorous thyroid tissues using RNAseqV2 data sets for thyroid cancer deposited on The Cancer Genome Atlas (TCGA, https://tcga-data.nci.nih.gov/tcga/) website. The results showed that SOSTDC1 expression was attenuated in thyroid cancer tissues compared to adjacent nontumorous thyroid tissues (Figure 1A). To further validate the expression of SOSTDC1 in thyroid cancer, we used real-time polymerase chain reaction (PCR) to determine the expression levels of SOSTDC1 in 22 pairs of tumors and their adjacent non-tumorous thyroid tissues. In agreement with the TCGA data, the expression levels of SOSTDC1 were significantly decreased in most thyroid tumor tissues in comparison with those in adjacent nontumorous thyroid tissues (Figure 1B). To study the protein expression of SOSTDC1, immunohistochemistry (IHC) was performed in 12 pairs of tumors and their adjacent

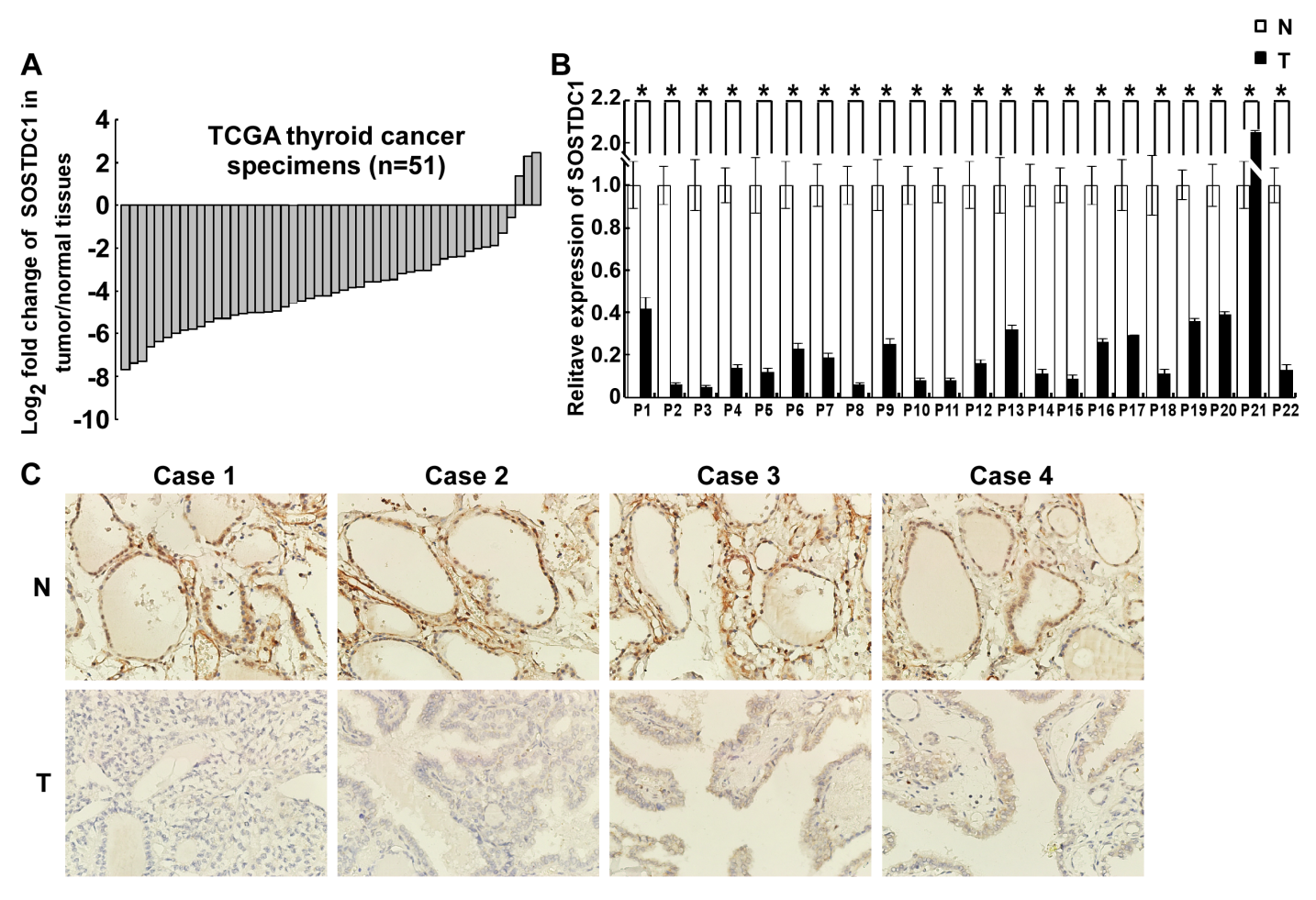

D

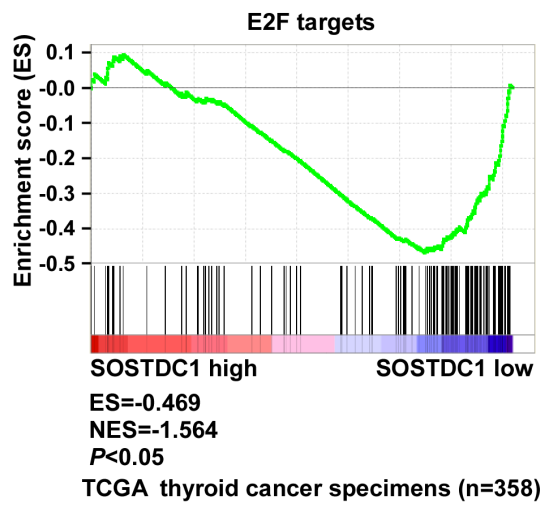

Figure 1: SOSTDC1 is clinically associated with the Rb-E2F pathway. A. The expression of SOSTDC1 in 51 pairs of primary tumors versus paired non-tumorous thyroid tissues using RNAseqV2 data sets deposited on TCGA website. B. Expression of SOSTDC1 in 22 paired tumors and adjacent non-tumorous thyroid tissues assessed by qRT-PCR. C. Expression of SOSTDC1 in 12 paired tumors and adjacent non-tumorous thyroid tissues assessed by IHC. D. GSEA plotting shows that SOSTDC1 expression is negatively correlated with a signature of genes responsive to E2F activation (TCGA dataset, $n=358$ ). 
non-tumorous thyroid tissues. The results showed that SOSTDC1 expression was significantly down-regulated in tumors as compared with their adjacent non-tumorous tissues (Figure 1C). These results imply that downregulation of SOSTDC1 may play a biologic role in human thyroid cancer disease processes.

To further investigate the functional influences and the involved pathways of down-regulated SOSTDC1 on thyroid cancer, we downloaded RNAseqV2 data sets for 358 cases of thyroid cancers from the TCGA data portal. We analyzed the fold differences in transcript abundance between low and high SOSTDC1 expression (median as cutoff) used MultiExperiment Viewer (MeV, http:// www.tm4.org/mev.html) software. Then the significantly differentially expressed genes were imported into the Ingenuity Pathway Analysis (IPA) software (Ingenuity Systems, http://www.ingenuity.com/) and analyzed. The analysis indicated that "cellular growth and proliferation" was the top biological function mediated by these differentially expressed genes identified in low and high SOSTDC1 expression samples. Furthermore, to find out the altered upstream regulators that could be responsible for the observed expression changes, we performed IPA Upstream Regulator Analysis (URA). URA could predict whether the upstream regulators are activated or inhibited based on the distinct up- and down-regulation pattern of the expressed genes, and determine which causal relationships previously reported in the literature are likely relevant for the biological mechanisms underlying the data. A Z-score $>2$ was considered to be activated, and Z-score $<-2$ was considered to be inhibited. URA analysis revealed that the transcriptional factor E2F, with a negative $\mathrm{Z}$-score, was one of the top predicted upstream regulators. As it is well known that E2F widely participates in the development of various cancers, we focused on studying whether E2F is involved in the effect of SOSTDC1 on the thyroid cancer cells (Figure 2).

We further carried out gene set enrichment analyses

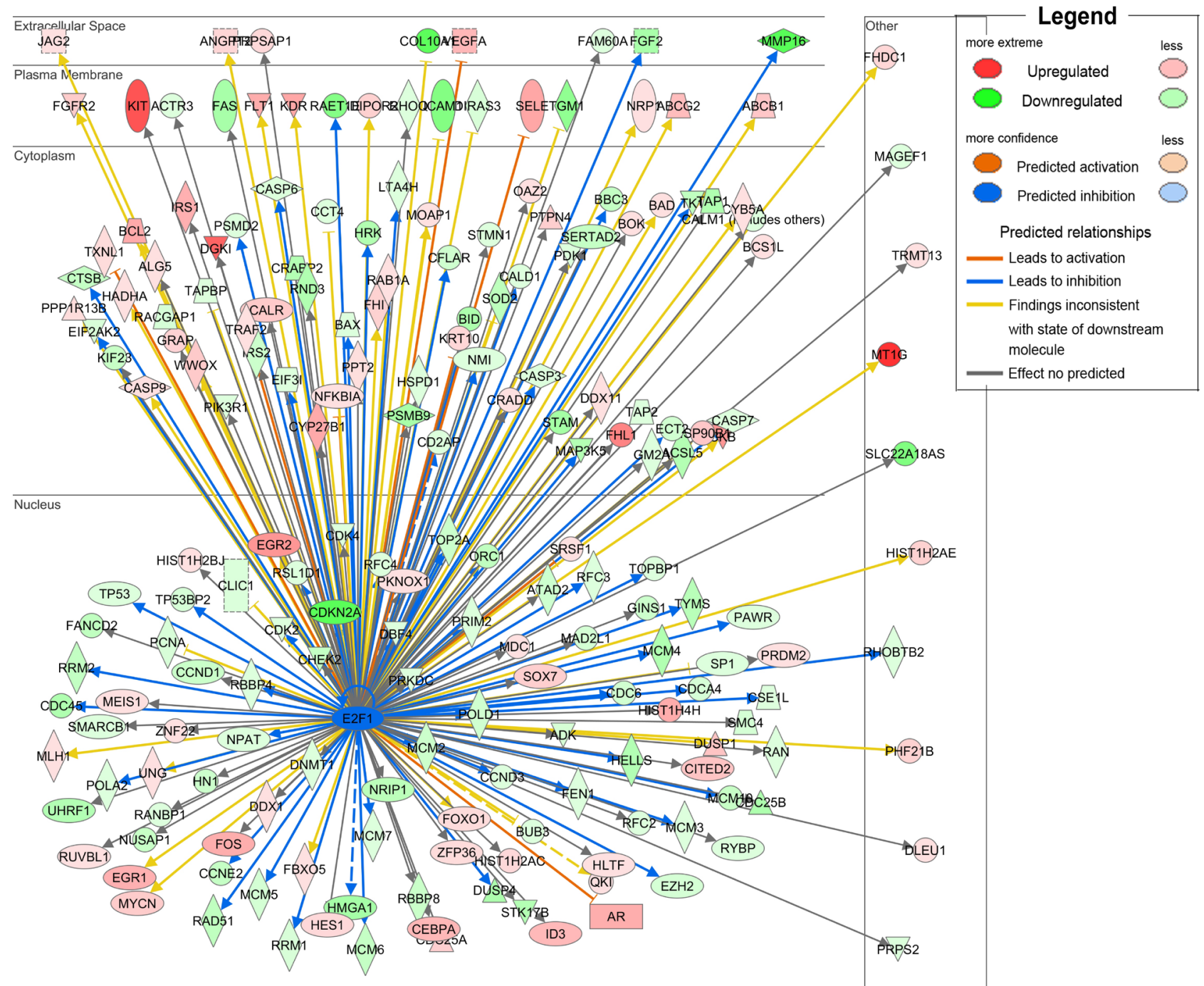

Figure 2: URA analysis reveals that the transcriptional factor E2F was the predicted upstream regulator responsible for the differential expression of genes identified in low and high SOSTDC1 expression samples. 
(GSEA, http://www.broadinstitute.org/gsea/index.jsp) to investigate whether E2F up-regulated genes are enriched among the thyroid cancer patients with low SOSTDC1 expression. As shown in Figure 1D, E2F signature was significantly enriched in low SOSTDC1 expression group, in comparison with high expression group, indicating preferential E2F activation in thyroid cancer specimens with low SOSTDC1 expression. Taken together, these results indicate that SOSTDC1 may inhibit thyroid tumorigenesis, at least partly, through regulating the $\mathrm{Rb}$ E2F pathway.

\section{SOSTDC1 is down-regulated in human thyroid cancer tissue}

To evaluate the clinical relevance of SOSTDC1 in thyroid cancer, we investigated the SOSTDC1 protein expression in 16 cases of goiters, 15 cases of adenomas, and 107 cases of thyroid cancers ( 26 cases FTC, 69 cases PTC, and 12 cases ATC) by immunohistochemistry. As shown in Figure 3, the expression level of SOSTDC1 protein in thyroid malignant lesions was significantly decreased as compared with that in benign lesions (goiters and adenomas). Interestingly, low expression of SOSTDC1 was detected in $88.8 \%$ thyroid cancer $(95 / 107)$, whereas SOSTDC1 is highly expressed in most goiter $(13 / 16)$ and adenoma specimens $(12 / 15)$. Of note, no significant difference was observed in the protein levels of SOSTDC1 in FTC, PTC, and ATC, implying that down-regulation of SOSTDC1 in thyroid cancers did not correlate with the grade of malignancy. To further evaluate the role of SOSTDC1 in thyroid cancer progression, we analyzed the correlation between SOSTDC1 expression and clinical features of patients. As summarized in Table 1 , there was significant correlation between SOSTDC1 expression and tumor-node-metastasis staging $(\mathrm{p}=0.008)$. In addition, SOSTDC1 expression was significantly correlated with the tumor size $(\mathrm{p}=0.038)$. The SOSTDC 1 expression levels did not differ significantly by age ( $\mathrm{p}$
$=0.161)$ and gender $(p=0.299)$. Taken together, these data suggested that SOSTDC1 is down-regulated in thyroid cancer and the altered expression of SOSTDC1 may play an important role in the process of cancer progression.

\section{Ectopic over-expression of SOSTDC1 inhibits proliferation and induces G1/S arrest in thyroid cancer cells}

To investigate the functional role of SOSTDC1 in thyroid cancer progression, we established two stably expressed SOSTDC1 thyroid cancer cell lines, K1/ SOSTDC1 and $8505 \mathrm{C} / \mathrm{SOSTDC} 1$ (Figure 4A). Next, we evaluated the cell viability by MTT assay. As shown in Figure 4B, ectopic expression of SOSTDC1 decreased the growth of both $\mathrm{K} 1$ and 8505 cells as compared with vector-control cells. Our data from colony formation assay demonstrate that SOSTDC1 over-expressing cells exhibited fewer colonies compared with control cells (Figure 4C and 4D). As shown by flow cytometry analysis in Figure 4E, the SOSTDC1 over-expression resulted in an elevated in G1-phase proportion and a reduced in G2/M phase proportion. These results suggest that SOSTDC1 plays an important role in cell cycle control for thyroid cancer cells. Moreover, we performed EdU assay to assess the influence of SOSTDC1 to the DNA synthesis. The results showed that the percentage of EdU-positive cells was lower on average in cells which were transfected with SOSTDC1 (Figure 4F and 4G). Collectively, these data indicate that SOSTDC1 inhibits proliferation of thyroid cancer cells.

\section{SOSTDC1 inhibits the expression of cyclin A2 and cyclin E2}

To understand specific mechanisms underlying the inhibitory effect of SOSTDC1 on thyroid cancer cell proliferation, we examined the protein expression level of cell cycle regulators by Western blot analysis.
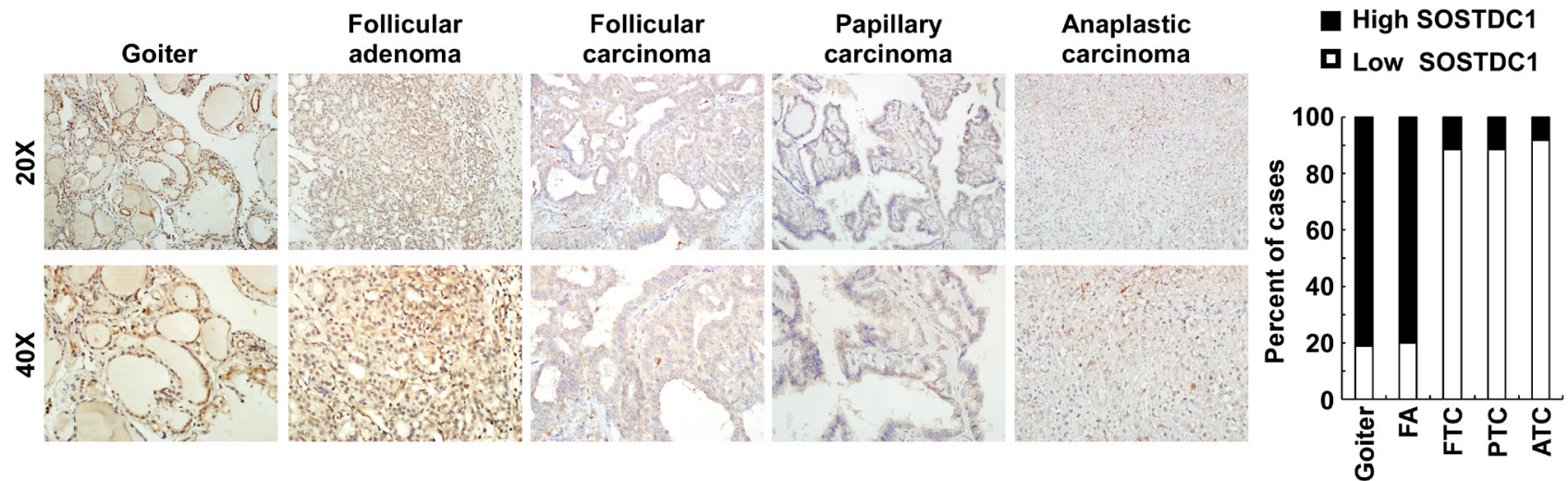

Figure 3: SOSTDC1 is down-regulated in human thyroid cancer tissue. A. Representative images of IHC assays on SOSTDC1 expression in thyroid lesions. B. Percentage of specimens showing low or high SOSTDC1 expression in thyroid lesions. 
A

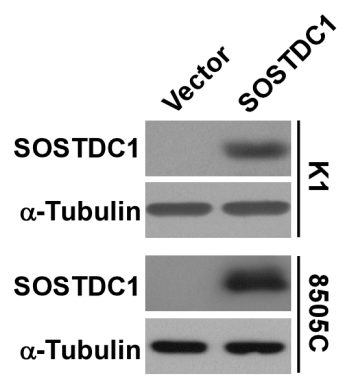

C

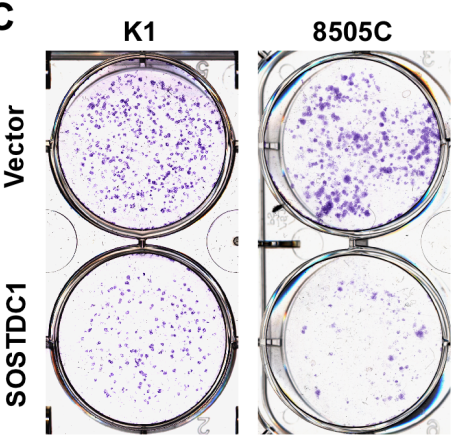

D

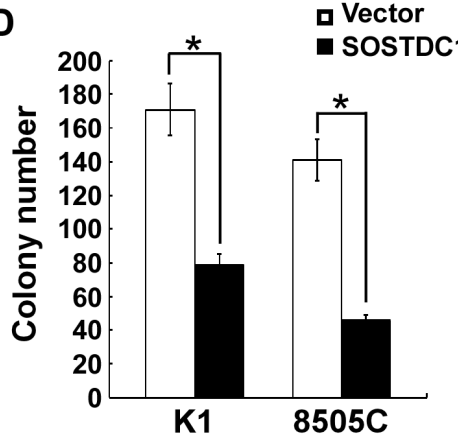

$\mathbf{F}$
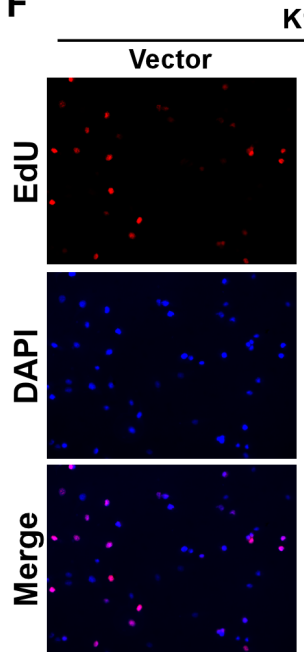

B
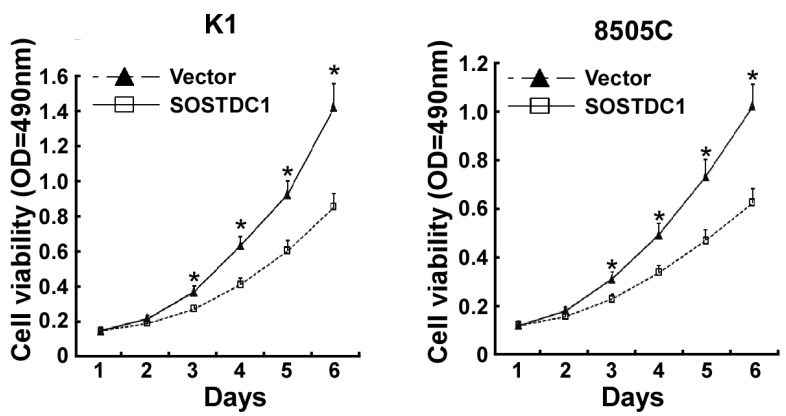

E
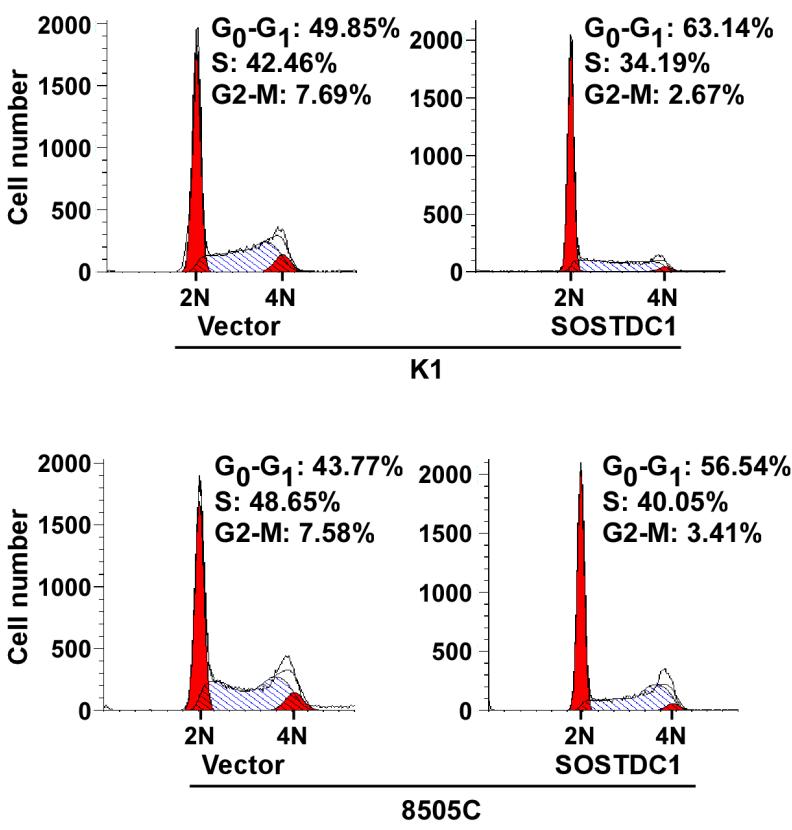

G

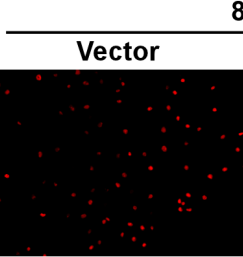

8505C
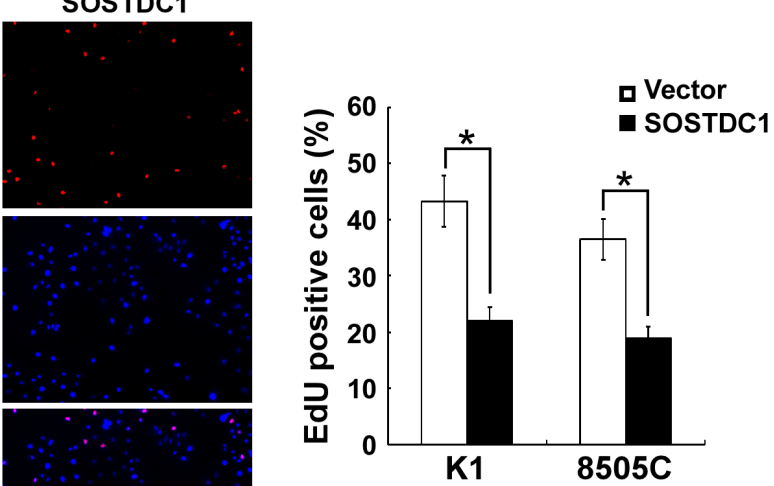
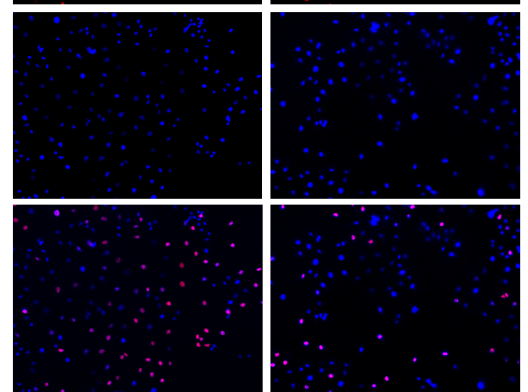

Figure 4: Ectopic over-expression of SOSTDC1 inhibits the proliferation of thyroid cancer cells. A.Protein expression of SOSTDC1 in indicated cells was analyzed by Western blotting assay. $\alpha$-tubulin was used as a loading control. B. MTT assay was conducted to investigate the effect of SOSTDC1 on the proliferation of indicated thyroid cancer cells at the indicated time points. C. and $\mathbf{D}$. Representative micrographs $\mathbf{C}$. and relative quantification $\mathbf{D}$. of colony formation assays of indicated cells. E. Flow-cytometric determination of proportion of indicated the studied cells in distinct cell-cycle phases. F. and $\mathbf{G}$. Representative images $\mathbf{F}$. and relative quantification G. of EdU incorporation assays. For B., D., and G., the data are reported as mean \pm SD of 3 independent experiments. ${ }^{*} P<$ 0.05 . 
As illustrated in Figure 5A, SOSTDC1 over-expression decreased the levels of cyclin A2 and cyclin E2 proteins in $\mathrm{K} 1$ and $8505 \mathrm{C}$ cells, but no changes in protein levels of cyclin B1, cyclin D1, cyclin D2, cyclin D3, cyclin E1, CDK2, CDK4, CDK6, p2 $1^{\text {Cip1 }}$, and $\mathrm{p} 27^{\text {Kip1 }}$ were observed. Moreover, over-expression of SOSTDC1 led to reduction of mRNA expression levels of cyclin A2 and cyclin E2 (Figure 5B). It is well known that Rb-E2F pathway is the main substrates of $\mathrm{CDK} /$ cyclin complexes, and the URA results show that $S O S T D C 1$ is clinically associated with the Rb-E2F pathway. So we further examined the effects of SOSTDC1 on pRb phosphorylation and E2F transcriptional activity. As expected, over-expression of SOSTDC1 decreased the phosphorylation of $\mathrm{pRb}$ at Ser608 and Ser807 residues (Figure 5C) and E2F transcriptional activity (Figure 5D). Taken together, these results indicate that SOSTDC1 suppresses proliferation through inhibiting the expression of cyclin A2 and cyclin $\mathrm{E} 2$ proteins, and the activity of Rb-E2F pathway.

\section{SOSTDC1 suppresses tumor growth in vivo}

To investigate the biological effect of ectopic overexpression of SOSTDC1 on tumor growth in vivo, we proceeded with the research through the establishment of a subcutaneous xenograft tumor model in nude mice. The growth curve revealed a dramatic decrease of tumor size in the group with SOSTDC1 over-expression (Figure 6A). As shown in Figure 6B and 6C, the tumor size and weight of the SOSTDC1 over-expression group was smaller than that of control group. IHC staining showed that ectopic over-expression of SOSTDC1 significantly inhibited the expression of Ki67, a well-established cell proliferation marker. In addition, consistent with the results of cell proliferation assays, cyclin A2 and cyclin E2 were also down-regulated following SOSTDC1 over-expression (Figure 6D). Therefore, SOSTDC1 inhibits the growth of thyroid cancer cells in vivo, likely by decreasing cyclin $\mathrm{A} 2$ and cyclin E2.
A

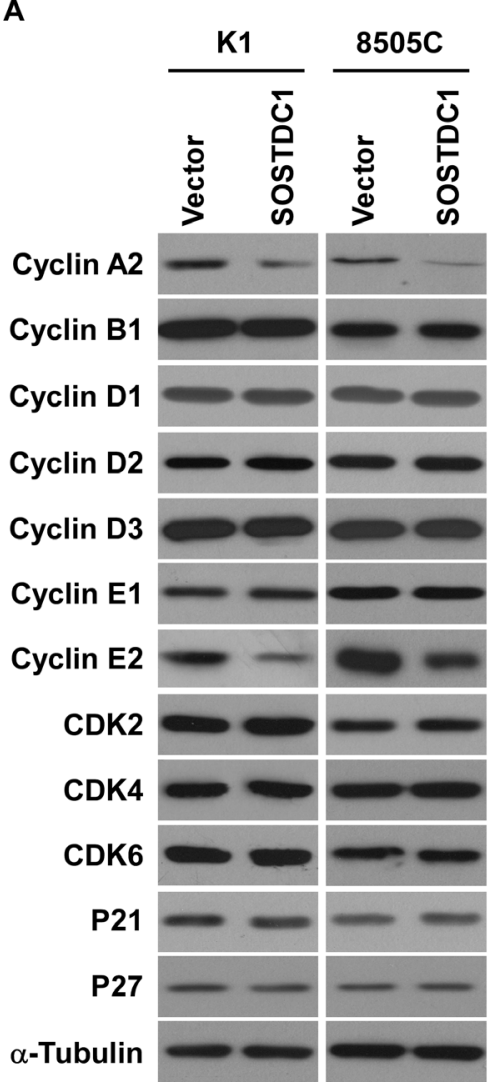

B
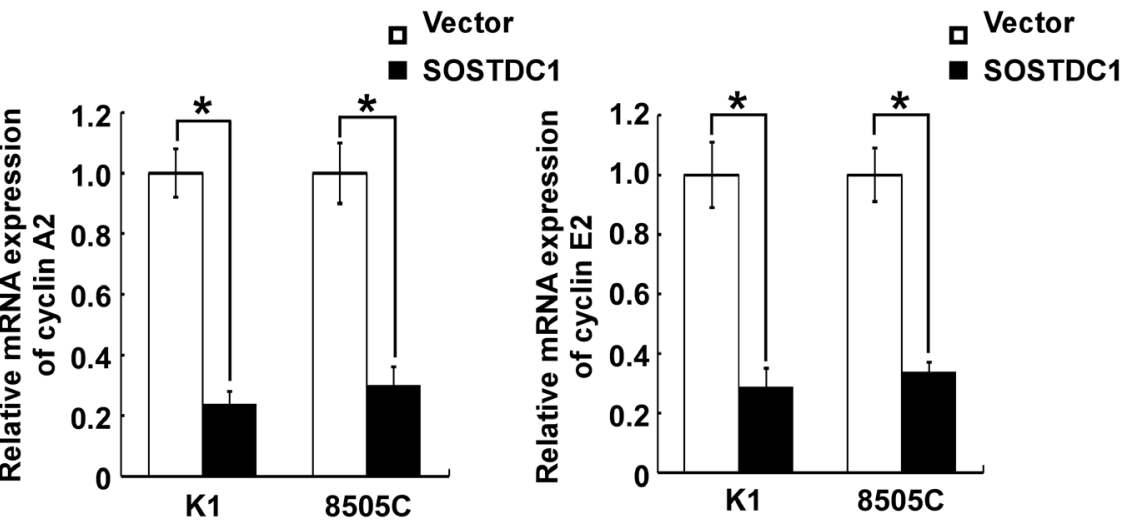

C

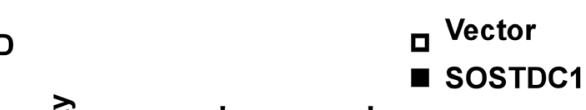

Figure 5: Ectopic over-expression of SOSTDC1 inhibits the expression of cyclinA2 and cyclin E2, and suppresses E2F transcriptional activity. A. Western blotting analysis was performed to detect the cell cycle regulator cyclin A2, cyclin B1, cyclin D1, cyclin D2, cyclin D3, cyclin E1, cyclin E2, CDK2, CDK4, CDK6, p2 $1^{\text {Cipl }}$, and p27 $7^{\mathrm{Kipl}}$ in indicated cells. $\alpha$-tubulin was used as a loading control.B. qRT-PCR was conducted to detect the mRNA expression levels of cyclin A2 and cyclin E2 in indicated cells. GAPDH was used as a house keeping gene control. C. Ectopic expression of SOSTDC1 in the studied cells significantly inhibited the phosphorylation of $\mathrm{pRb}$ at Ser608 and Ser807 residues. $\alpha$-tubulin served as the sample loading control. D. Over-expression of SOSTDC1 attenuates E2F transcriptional activity using E2F-luc reporter assay. For B. and D., results derived from three independent experiments are expressed as mean \pm SD. $* \mathrm{P}<0.05$. 


\section{DISCUSSION}

The current study demonstrated that SOSTDC1 expression was significantly reduced in thyroid cancer. Moreover, ectopic over-expression of SOSTDC1 led to down-regulation of cyclin A2 and cyclin E2, which subsequently inhibit thyroid cancer cell proliferation in vitro and in vivo. Our results underline a fundamental role of SOSTDC1 as a tumor suppressor in thyroid cancer.

An increasing number of studies have demonstrated that SOSTDC1 is dysregulated and acts as a tumor suppressor in some kinds of human cancers. For example, it was previously indicated that SOSTDC1 is significantly down-regulated in clear cell renal carcinoma and overexpression of SOSTDC1 inhibits its proliferation through suppressing BMP-7-induced phosphorylation of Smad-1, -5 , and -8 , as well as Wnt-3a signaling [10]. Moreover, Blish KR et al. confirmed that loss of heterozygosity for SOSTDC1 has been identified in adult and pediatric renal tumors [18]. In Wilms tumor, SOSTDC1 has been identified as a candidate tumor suppressor gene, which may have a role in the progression of tumorigenesis [11]. Recently, it was demonstrated that SOSTDC1 protein expression was down-regulated in gastric tumors, and ectopic over-expression in gastric cancer cells inhibits their tumorigenic properties [13]. Indeed, Barderas et al. reported that SOSTDC1 was up-regulated in highly metastatic KM12SM colorectal cancer cells, and silencing of SOSTDC1 caused a significant decrease in migration, invasion, and metastasis [19]. In our present study, for the first time SOSTDC1 was found to be down-regulated and functioned as a tumor suppressor gene through inhibiting cell proliferation in thyroid cancer. However, the mechanisms involved in down-regulation of SOSTDC1 in thyroid cancer are largely unknown. Recently, downregulation of SOSTDC1 expression by epigenetic mechanisms, such as methylation, has been documented in cancer. Gopisetty et al. observed that SOSTDC1 methylation can inhibit SOSTDC1 promoter activity and suppress gene expression in gastric cancer [13]. In breast cancer, methylation also participated in the downregulation of SOSTDC1. Akhilesh et al. showed that the transcriptional repressor E4BP4 epigenetically repressed SOSTDC1 expression in breast cancer cells [20]. Thus, it is likely that epigenetic mechanisms might also play a role in down-regulation of SOSTDC1 in thyroid cancer.

We also further explored the potential mechanism by which SOSTDC1 suppresses thyroid cancer cell proliferation. The results showed that over-expression of SOSTDC1 in thyroid cancer cells resulted in dysregulating the expression of critical cell cycle regulators, cyclin A2 and cyclin E2 in vitro and vivo. Moreover, consistent with the results of biologic analysis, we found that Rb-E2F pathway also involved in the antiproliferative role.

Cyclins are activators of specific serine/threonine protein kinases, which can promote the cell cycle process. Cyclin A2, which is particular in the cyclin family, activates two different cyclin-dependent kinases (CDKs) and promotes both $\mathrm{G} 1 / \mathrm{S}$ and $\mathrm{G} 2 / \mathrm{M}$ phase transitions [21]. Consistent with its role as a key cell cycle regulator, over-expression of cyclin A2 is found in many cancers, including anaplastic thyroid carcinoma [22]. In addition, over-expression of cyclin A2 in cancers is associated with enhanced cellular proliferation and predicted a poor prognosis [23]. Cyclin E2, which can bind to Cdc2related kinase (CDK2), plays a critical role for $\mathrm{G} 1 / \mathrm{S}$ transition. It aberrantly expresses in human cancers [24], and its over-expression is associated with proliferation in various cancers. For example, the levels of cyclin E2 was significantly elevated in human breast cancers [25], and decrease of cyclin E2 severely attenuates the estrogeninduced breast cancer cell proliferation [26]. Moreover, the high expression level of Cyclin E2 was related to a poor prognosis in ER-positive breast cancer [27, 28]. Cyclin E2 is also over-expressed in several small lung cancer cell lines [24], and ectopic over-expression of cyclin E2 reversed the G0/G1 cell cycle arrest induced by miR-25 down-regulation [29]. Similar with the previous studies, our data demonstrated that decreased cyclin A2 and cyclin E2 were involved in the anti-proliferative role of SOSTDC1 in thyroid cancer.

To further address the molecular mechanisms involved in SOSTDC1-mediated inhibition of proliferation, Rb-E2F pathway was selected for study. Because it is well known that Rb-E2F pathway is the main substrate of CDK/ cyclin complexes, and the URA results show that SOSTDC1 is clinically associated with the RbE2F pathway. Emerging evidence has shown that $\mathrm{Rb}$ is a tumor suppressor and plays a critical role in regulating G1/S transition and cell proliferation. Rb exerts its growth regulatory properties, in large part by repressing the transcriptional activity of the E2F transcription factors. $\mathrm{Rb}-\mathrm{E} 2 \mathrm{~F}$ pathway plays an important role in regulating tumor cell proliferation [30]. However, little is known about the function of Rb-E2F pathway in thyroid cancer. Our study demonstrated that over-expression of SOSTDC1 significantly inhibited the phosphorylation of $\mathrm{pRb}$ and E2F transcriptional activity.

In conclusion, our data demonstrate that SOSTDC1 is down-regulated in thyroid tumor tissues and inhibits thyroid cancer cell proliferation through modulating cyclin A2 and cyclin E2. Given these findings, SOSTDC1 might be a potential therapeutic target in the treatment of thyroid cancer. 
Table 1: Association between clinicopathologic parameters and level of SOSTDC1 protein expression in thyroid cancer patients

\begin{tabular}{|c|c|c|c|}
\hline \multirow[t]{2}{*}{ Clinicopathologic variables } & \multicolumn{2}{|c|}{ SOSTDC1 } & \multirow[t]{2}{*}{$P$ value } \\
\hline & Low & High & \\
\hline \multicolumn{4}{|l|}{ Age } \\
\hline$<45 \mathrm{yr}$ & 51 & 9 & 0.161 \\
\hline$\geq 45 \mathrm{yr}$ & 44 & 3 & \\
\hline \multicolumn{4}{|l|}{ Gender } \\
\hline Male & 22 & 5 & 0.299 \\
\hline Female & 73 & 7 & \\
\hline \multicolumn{4}{|l|}{ TNM stage } \\
\hline I and II & 49 & 11 & 0.008 \\
\hline III and IV & 46 & 1 & \\
\hline \multicolumn{4}{|l|}{$\mathrm{T}$ classification } \\
\hline $\mathrm{T} 1$ and $\mathrm{T} 2$ & 53 & 11 & 0.038 \\
\hline $\mathrm{T} 3$ and $\mathrm{T} 4$ & 42 & 1 & \\
\hline \multicolumn{4}{|l|}{$\mathrm{N}$ classification } \\
\hline No & 46 & 8 & 0.083 \\
\hline N1 & 49 & 4 & \\
\hline
\end{tabular}

SOSTDC1, sclerostin domain containing protein 1.

A

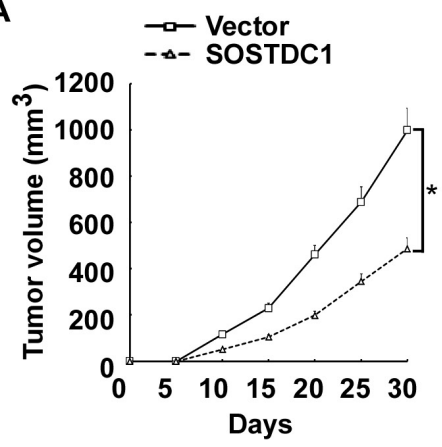

D

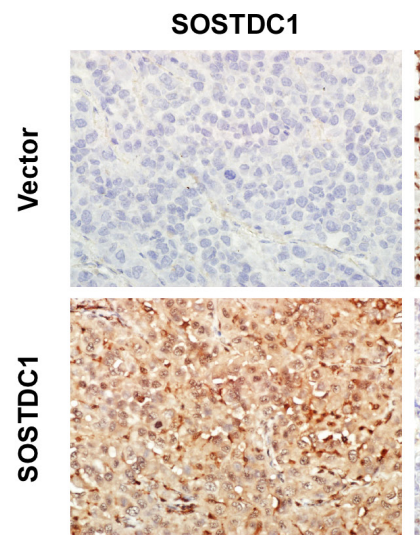

B

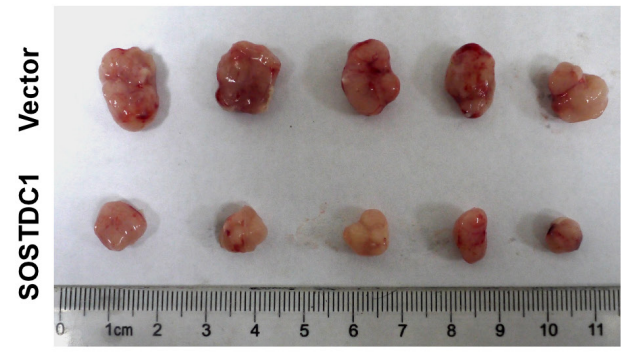

C

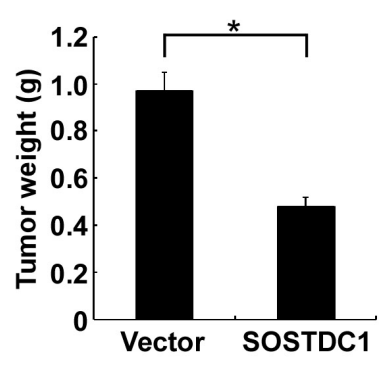

Figure 6: SOSTDC1 suppresses tumor growth in vivo. A. Quantitative analysis of tumor volumes. B. Representative image of subcutaneous tumors isolated from nude mice. C. Quantitative analysis of tumor weights. The indicated tumor volumes and weights represent the mean \pm SD of five animals in each group. D. Representative images of IHC assays on SOSTDC1, cyclin A2, cyclin E2 and Ki67 expression in resected tumor specimens. ${ }^{*} \mathrm{p}<0.05$. 


\section{MATERIALS AND METHODS}

\section{Cell culture}

Human thyroid cancer cell lines, K1 and 8505C cells were obtained from the European Collection of Cell Cultures (ECACC, Salisbury, United Kingdom) and maintained in DMEM with 10\% FBS.

\section{Vectors and retroviral infection}

A SOSTDC 1 construct was generated by subcloning polymerase chain reaction (PCR)-amplified full-length human SOSTDC1 cDNA into pQCXIP (Clontech, Palo Alto, CA). For retroviral transduction, Pt67 (Clontech, Palo Alto, CA) was incubated with a precipitated mixture of Lipofectamine 2000 (Life Technologies, Gaithersburg, $\mathrm{MD})$ and pQCXIP-SOSTDC1 plasmid for $24 \mathrm{~h}$ [31]. Stable cell lines were selected by treatment with $0.5 \mu \mathrm{g} / \mathrm{ml}$ puromycin for 10 days, beginning 48 hours after infection.

\section{Patients and tissue specimens}

A total of 138 cases of paraffin-embedded thyroid tissue ( 26 cases of FTC, 69 cases of PTC, and 12 cases of ATC, 15 cases of adenoma samples, and 16 cases of goiters) and 22 pairs of human thyroid cancer tissues and their adjacent non-cancerous thyroid tissues were obtained from the First Affiliated Hospital of Sun Yat-sen University between 2008 to 2013, with the approval of the Institutional Research Ethics Committee. The pathology of all tissue parts was confirmed by two pathologists. The 22 pairs of tissue specimens were collected, frozen, and stored in liquid nitrogen until used.

\section{MTT assay}

Cell growth was determined using a MTT assay. The cells were seeded at a density of $5 \times 10^{3}$ cells per well in 96-well plates. Then at 1, 2, 3, 4, 5 and 6 days, $20 \mu$ MTT (Sigma-Aldrich, St. Louis, MO) was added to each well and incubated for $4 \mathrm{~h}$. The culture medium was removed and $200 \mu 1$ dimethyl sulfoxide (DMSO) (Amresco, Solon, Ohio) was added to each well. The plates were then shaken for 30 minutes, and the optical density (OD) at $490 \mathrm{~nm}$ was measured using an ELISA reader. Each experiment was performed in triplicate.

\section{Colony formation assay}

For the colony formation assay, cells were plated at 500 per well into 6-well plates. The cells were allowed to grow 10 days and stained with crystal violet. The plates were photographed and the numbers of colonies formed by indicated cells were quantified using the Quantity One software package (Bio-Rad, Hercules, CA). Each experiment was repeated three times.

\section{Cell cycle analysis}

For cell cycle analysis, cells were harvested by trypsinization and washed twice with ice-cold phosphatebuffered saline (PBS). The cells were then fixed in $75 \%$ ethanol, treated with RNase A (Sigma-Aldrich, St. Louis, MO), followed by incubation with propidium iodide (Sigma-Aldrich, St. Louis, MO). Cell cycle analysis was determined using a flow cytometer (Beckman-Coulter, Hialeah, FL).

\section{5-ethynyl-2'-deoxyuridine (Edu) incorporation assay}

To determine the DNA synthesis, the Cell Light EdU DNA imaging kit (RiboBio Co., Guangzhou, China) was used. In short, cells were seeded in 24-well plates and exposed to EdU for $2 \mathrm{~h}$. Then they were fixed in $4 \%$ paraformaldehyde and permeabilized in $0.5 \%$ Triton $\mathrm{X}-100$. Images were taken using a fluorescent microscope at $488 \mathrm{~nm}$ excitation. Each experiment was repeated independently thrice.

\section{Western blotting}

Western blotting was performed according to a standard method as described previously [32]. Antibodies for immunoblotting were as follows: anti-SOSTDC1, anti-cyclin B1, anti-cyclin D3 (Abcam, Cambridge, $\mathrm{MA}$, anti-cyclin A2, anti-cyclin D1, anti-cyclin E1, antiCDK4, anti-CDK6 (Epitomics, Burlingame, California), anti-cyclin D2, anti-CDK2 (BD Pharmingen, San Diego, CA), anti-cyclin E2, anti-p2 $1^{\text {Cip1 }}$, anti-p27 $7^{\text {Kip1 } 1}$, anti-p-pRb Ser608, anti-p-pRb Ser807, anti-pRb (Cell Signaling, Beverly, MA) and anti- $\alpha$-tubulin (Sigma-Aldrich, St. Louis, Missouri).

\section{RNA extraction and real-time reverse transcription-polymerase chain reaction}

Total RNA from tissues was extracted by TRizol reagent (Life Technologies, Gaithersburg, $\mathrm{MD}$ ), and reverse transcription (RT) reactions and real-time polymerase chain reaction (PCR) were performed as described previously [33]. The following primers were used: SOSTDC1 forward, 5'CACGTTGAATCAAGCCAGAA-3' and reverse, 5'GATGTATTTGGTGGAACGCA-3'; cyclin A2 forward, 
5'- CAGAAAACCATTGGTCCCTC-3' and reverse, 5' -CACTCACTGGCTTTTCATCTTC-3'; cyclin E2 forward, 5'- ACCTCATTATTCATTGCTTCCAA-3' and reverse, 5'-TCTTCACTGCAAGCACCATC-3'; and GAPDH forward, 5'-GACTCATGACCACAGTCCATGC-3' and reverse, 5'-AGAGGCAGGGATGATGTTCTG-3'.

\section{Luciferase reporter assay}

Dual-Luciferase reporter assays (Promega, Madison, Wisconsin) were performed according to the manufacturer's instructions as previously described [33]. Briefly, $\mathrm{K} 1$ and $8505 \mathrm{C}$ cells were seeded in triplicate in 24-well plates and allowed to settle for $24 \mathrm{~h}$. Cotransfection of pE2F-TA-Luc plasmid (Clontech, San Francisco, CA) and $1 \mathrm{ng}$ pRL-TK Renilla was performed using Lipofectamine 2000 Reagent (Life Technologies, Gaithersburg, MD) according to the manufacturer's protocol. Thirty six hours after transfection, the cells were harvested and lysed, and the luciferase activities were assessed. The firefly luciferase activities were normalized to Renilla luciferase activities. Three independent experiments were performed.

\section{In vivo experiments}

Five female BALB/c mice (4 weeks old) were used to assess the effect of SOSTDC1 on tumor growth in vivo. Briefly, $5 \times 10^{6} \mathrm{~K} 1 / \mathrm{SOSTDC} 1$ cells were injected subcutaneously into the left dorsal flank of each mouse, and the right side was inoculated with $5 \times 10^{6} \mathrm{~K} 1 /$ Vector as a control. Tumor size was measured every 5 days, and the tumor volume was estimated. Thirty days after the injection, the mice were euthanized, and the tumors were removed and weighed. Then the excised tumors were fixed in $10 \%$ formalin for $24 \mathrm{~h}$, embedded in paraffin wax and serially sectioned for $5 \mu \mathrm{m}$ slides for immunohistochemistry staining. All experiments involving the use of animals were conducted in accordance with the recommendations in the Guide for the Care and Use of Laboratory Animals of the National Institutes of Health. The protocol was approved by the Institutional Animal Care and Use Committee of Sun Yat-sen University.

\section{Immunohistochemistry}

Sections were deparaffinized, rehydrated, subjected to antigen retrieval and blocked. Then the tissues were incubated with the following antibodies: anti-SOSTDC1, anti-cyclin E2 (Abcam, Cambridge, MA), anti-cyclin A2 (Cell Signaling, Beverly, MA), and anti-Ki67 (SigmaAldrich, St. Louis, Missouri). The secondary antibodies were used. The immunohistochemical staining was evaluated in accordance with our previous report [34].
In brief, the proportion of tumor cells was graded as follows: 0 (no positive tumor cells), $1(<10 \%$ positive tumor cells), 2 (10-50\% positive tumor cells), and 3 (> $50 \%$ positive tumor cells). The intensity of staining was determined as: 0 (no staining); 1 (weak staining $=$ light yellow), 2 (moderate staining $=$ yellow brown), and 3 (strong staining $=$ brown). The staining index $(\mathrm{SI})$ was calculated as staining intensity score $\times$ proportion of positive tumor cells, resulting in scores of $0,1,2,3,4,6$ and 9. An SI score of $\geq 4$ was used to define tumors with high expression, and $\leq 3$ as tumors with low expression.

\section{Bioinformatic analysis}

We downloaded RNAseqV2 data of 51 pairs of thyroid tumors and their adjacent non-tumorous thyroid tissues, and 358 cases of thyroid cancers from The Cancer Genome Atlas (TCGA) website (https:// tcga-data.nci.nih.gov/tcga) [35]. The fold differences in transcript abundance were compared by paired t test, using MultiExperiment Viewer (MeV) software (version 4.9, http://www.tm4.org/). Ingenuity Pathway Analysis (IPA) was conducted to identify the biological function mediated by these differentially expressed genes identified in low and high SOSTDC1 expression samples. Then IPA Upstream Regulator Analysis (URA) was performed to find out the altered upstream regulators that could be responsible for the observed expression changes. Gene Set Enrichment Analysis (GSEA) was carried out using GSEA 2.0 .14 (http://www.broadinstitute.org/gsea/). The E2F target gene set was compiled from a review by Bracken et al. [36].

\section{Statistical analysis}

All data are presented as means \pm standard deviation (SD) of at least three independent experiments. Statistical analysis was performed using the SPSS17.0 software (SPSS Inc., Chicago, IL) and student t-test was used to compare the differences between two groups. A value of $P$ $<0.05$ was considered statistically significant.

\section{CONFLICTS OF INTEREST}

The authors declare no conflicts of interest.

\section{GRANT SUPPORT}

This research was supported by grants from the Doctoral Fund of Ministry of Education, China (No. 20130171110067); Guangzhou Municipal Science and Technology special fund (No. 1346000270); Industrial Technology Research and Development funding projects, Guangdong Province (No. 2012A030400006); 2014 Technology special projects of Guangzhou (No. 2014Y2- 
00127); the Natural Science Foundation of China (No. 81370076, 81572624 and 81572623); Key Medical Laboratory of Guangdong Province.

\section{REFERENCES}

1. Siegel R, Naishadham D and Jemal A. Cancer statistics, 2013. CA Cancer J Clin. 2013; 63:11-30.

2. Russo D, Damante G, Puxeddu E, Durante C and Filetti S. Epigenetics of thyroid cancer and novel therapeutic targets. J Mol Endocrinol. 2011; 46:R73-81.

3. Nikiforov YE and Nikiforova MN. Molecular genetics and diagnosis of thyroid cancer. Nat Rev Endocrinol. 2011; 7:569-580.

4. Lintern KB, Guidato S, Rowe A, Saldanha JW and Itasaki $\mathrm{N}$. Characterization of wise protein and its molecular mechanism to interact with both Wnt and BMP signals. J Biol Chem. 2009; 284:23159-23168.

5. Kassai Y, Munne P, Hotta Y, Penttila E, Kavanagh K, Ohbayashi N, Takada S, Thesleff I, Jernvall J and Itoh N. Regulation of mammalian tooth cusp patterning by ectodin. Science. 2005; 309:2067-2070.

6. Beaudoin GM, 3rd, Sisk JM, Coulombe PA and Thompson CC. Hairless triggers reactivation of hair growth by promoting Wnt signaling. Proc Natl Acad Sci U S A. 2005; 102:14653-14658.

7. Collette NM, Yee CS, Murugesh D, Sebastian A, Taher L, Gale NW, Economides AN, Harland RM and Loots GG. Sost and its paralog Sostdc1 coordinate digit number in a Gli3-dependent manner. Dev Biol. 2013; 383:90-105.

8. Shigetani Y, Howard S, Guidato S, Furushima K, Abe T and Itasaki N. Wise promotes coalescence of cells of neural crest and placode origins in the trigeminal region during head development. Dev Biol. 2008; 319:346-358.

9. Clausen KA, Blish KR, Birse CE, Triplette MA, Kute TE, Russell GB, D'Agostino RB, Jr., Miller LD, Torti FM and Torti SV. SOSTDC1 differentially modulates Smad and beta-catenin activation and is down-regulated in breast cancer. Breast Cancer Res Treat. 2011; 129:737-746.

10. Blish KR, Wang W, Willingham MC, Du W, Birse CE, Krishnan SR, Brown JC, Hawkins GA, Garvin AJ, D'Agostino RB, Jr., Torti FM and Torti SV. A human bone morphogenetic protein antagonist is down-regulated in renal cancer. Mol Biol Cell. 2008; 19:457-464.

11. Ohshima J, Haruta M, Arai Y, Kasai F, Fujiwara Y, Ariga T, Okita H, Fukuzawa M, Hata J, Horie H and Kaneko Y. Two candidate tumor suppressor genes, MEOX2 and SOSTDC1, identified in a $7 \mathrm{p} 21$ homozygous deletion region in a Wilms tumor. Genes Chromosomes Cancer. 2009; 48:1037-1050.

12. Rajkumar T, Vijayalakshmi N, Gopal G, Sabitha K, Shirley S, Raja UM and Ramakrishnan SA. Identification and validation of genes involved in gastric tumorigenesis. Cancer Cell Int. 2010; 10:45.

13. Gopal G, Raja UM, Shirley S, Rajalekshmi KR and
Rajkumar T. SOSTDC1 down-regulation of expression involves $\mathrm{CpG}$ methylation and is a potential prognostic marker in gastric cancer. Cancer Genet. 2013; 206:174-182.

14. Manning AL and Dyson NJ. RB: mitotic implications of a tumour suppressor. Nat Rev Cancer. 2012; 12:220-226.

15. Weinberg RA. The retinoblastoma protein and cell cycle control. Cell. 1995; 81:323-330.

16. Gordon GM and $\mathrm{Du}$ W. Conserved RB functions in development and tumor suppression. Protein Cell. 2011; 2:864-878.

17. Singh S, Johnson J and Chellappan S. Small molecule regulators of $\mathrm{Rb}-\mathrm{E} 2 \mathrm{~F}$ pathway as modulators of transcription. Biochim Biophys Acta. 2010; 1799:788-794.

18. Blish KR, Clausen KA, Hawkins GA, Garvin AJ, Willingham MC, Turner JC, Torti FM and Torti SV. Loss of heterozygosity and SOSTDC1 in adult and pediatric renal tumors. J Exp Clin Cancer Res. 2010; 29:147.

19. Barderas R, Mendes M, Torres S, Bartolome RA, LopezLucendo M, Villar-Vazquez R, Pelaez-Garcia A, Fuente $\mathrm{E}$, Bonilla F and Casal JI. In-depth characterization of the secretome of colorectal cancer metastatic cells identifies key proteins in cell adhesion, migration, and invasion. Mol Cell Proteomics. 2013; 12:1602-1620.

20. Rawat A, Gopisetty G and Thangarajan R. E4BP4 is a repressor of epigenetically regulated SOSTDC1 expression in breast cancer cells. Cell Oncol (Dordr). 2014; 37:409419.

21. Gong D, Pomerening JR, Myers JW, Gustavsson C, Jones JT, Hahn AT, Meyer T and Ferrell JE, Jr. Cyclin A2 regulates nuclear-envelope breakdown and the nuclear accumulation of cyclin B1. Curr Biol. 2007; 17:85-91.

22. Zhong WB, Hsu SP, Ho PY, Liang YC, Chang TC and Lee WS. Lovastatin inhibits proliferation of anaplastic thyroid cancer cells through up-regulation of $\mathrm{p} 27$ by interfering with the Rho/ROCK-mediated pathway. Biochem Pharmacol. 2011; 82:1663-1672.

23. Yasmeen A, Berdel WE, Serve H and Muller-Tidow C. E- and A-type cyclins as markers for cancer diagnosis and prognosis. Expert Rev Mol Diagn. 2003; 3:617-633.

24. Gudas JM, Payton M, Thukral S, Chen E, Bass M, Robinson $\mathrm{MO}$ and Coats S. Cyclin E2, a novel G1 cyclin that binds Cdk2 and is aberrantly expressed in human cancers. Mol Cell Biol. 1999; 19:612-622.

25. Payton M, Scully S, Chung G and Coats S. Deregulation of cyclin E2 expression and associated kinase activity in primary breast tumors. Oncogene. 2002; 21:8529-8534.

26. Caldon CE, Sergio CM, Schutte J, Boersma MN, Sutherland RL, Carroll JS and Musgrove EA. Estrogen regulation of cyclin E2 requires cyclin D1 but not c-Myc. Mol Cell Biol. 2009; 29:4623-4639.

27. Desmedt C, Ouriaghli FE, Durbecq V, Soree A, Colozza MA, Azambuja E, Paesmans M, Larsimont D, Buyse M, Harris A, Piccart M, Martiat P and Sotiriou C. Impact of cyclins E, neutrophil elastase and proteinase 3 expression 
levels on clinical outcome in primary breast cancer patients. Int J Cancer. 2006; 119:2539-2545.

28. Sieuwerts AM, Look MP, Meijer-van Gelder ME, Timmermans M, Trapman AM, Garcia RR, Arnold M, Goedheer AJ, de Weerd V, Portengen H, Klijn JG and Foekens JA. Which cyclin E prevails as prognostic marker for breast cancer? Results from a retrospective study involving 635 lymph node-negative breast cancer patients. Clin Cancer Res. 2006; 12:3319-3328.

29. Zhao Z, Liu J, Wang C, Wang Y, Jiang Y and Guo M. MicroRNA-25 regulates small cell lung cancer cell development and cell cycle through cyclin E2. Int J Clin Exp Pathol. 2014; 7:7726-7734.

30. Gordon GM and Du W. Targeting Rb inactivation in cancers by synthetic lethality. Am J Cancer Res. 2011; 1:773-786.

31. Yamauchi K, Yang M, Hayashi K, Jiang P, Yamamoto $\mathrm{N}$, Tsuchiya H, Tomita K, Moossa AR, Bouvet M and Hoffman RM. Induction of cancer metastasis by cyclophosphamide pretreatment of host mice: an opposite effect of chemotherapy. Cancer Res. 2008; 68:516-520.

32. Guan H, Cai J, Zhang N, Wu J, Yuan J, Li J and Li M. $\mathrm{Sp} 1$ is upregulated in human glioma, promotes MMP-2mediated cell invasion and predicts poor clinical outcome. Int J Cancer. 2012; 130:593-601.

33. Guan H, Wei G, Wu J, Fang D, Liao Z, Xiao H, Li M and Li Y. Down-regulation of miR-218-2 and its host gene SLIT3 cooperate to promote invasion and progression of thyroid cancer. J Clin Endocrinol Metab. 2013; 98:E1334-1344.

34. Guan H, Liang W, Liu J, Wei G, Li H, Xiu L, Xiao H and Li Y. Transmembrane Protease Serine 4 Promotes Thyroid Cancer Proliferation via CREB Phosphorylation. Thyroid. 2014.

35. Cancer Genome Atlas Research N. Comprehensive genomic characterization defines human glioblastoma genes and core pathways. Nature. 2008; 455:1061-1068.

36. Bracken AP, Ciro M, Cocito A and Helin K. E2F target genes: unraveling the biology. Trends Biochem Sci. 2004; 29:409-417. 